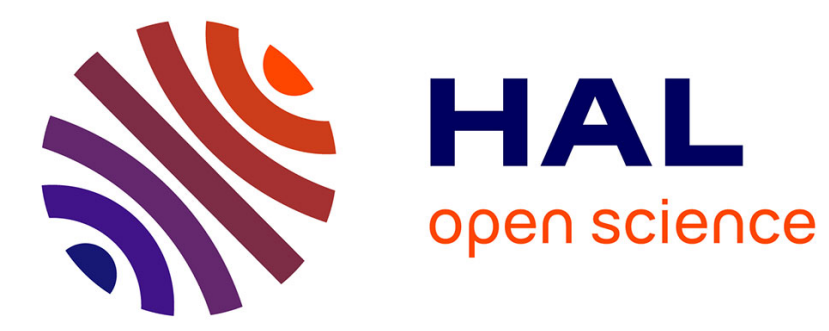

\title{
Enhancement of Nonlinear Effects at the Degenerate Band Edge of 2D Photonic Crystals
}

\author{
Magali Astic, Philippe Delaye, Robert Frey, Gérald Roosen
}

\section{To cite this version:}

Magali Astic, Philippe Delaye, Robert Frey, Gérald Roosen. Enhancement of Nonlinear Effects at the Degenerate Band Edge of 2D Photonic Crystals. Physical Review E : Statistical, Nonlinear, and Soft Matter Physics, 2009, 79 (5), pp.056608. 10.1103/PhysRevE.79.056608 . hal-00554113

\section{HAL Id: hal-00554113 \\ https://hal-iogs.archives-ouvertes.fr/hal-00554113}

Submitted on 10 Jan 2011

HAL is a multi-disciplinary open access archive for the deposit and dissemination of scientific research documents, whether they are published or not. The documents may come from teaching and research institutions in France or abroad, or from public or private research centers.
L'archive ouverte pluridisciplinaire HAL, est destinée au dépôt et à la diffusion de documents scientifiques de niveau recherche, publiés ou non, émanant des établissements d'enseignement et de recherche français ou étrangers, des laboratoires publics ou privés. 


\title{
Enhancement of Nonlinear Effects at the Degenerate Band Edge of 2D Photonic Crystals
}

\author{
M. Astic, Ph. Delaye, R. Frey, and G. Roosen \\ Laboratoire Charles Fabry de l'Institut d'Optique, Centre National de la Recherche \\ Scientifique et Université Paris-Sud, \\ Campus Polytechnique, RD 128, 91128 Palaiseau cedex, France
}

\begin{abstract}
The ability of 2D photonic crystals (PC) for high enhancements of nonlinear processes is analyzed in the case of a degenerate band edge when two symmetrical diffracted beams are generated by Bragg diffraction in the 2D-PC. Calculations are performed using the very simple Bragg coupled wave theory which only involves three coupled waves (the incident wave and the two diffracted waves) for the linear interaction. The validity of the approximation is proved for wavelengths lying at the neighbourhood of the band edges of 2D-PC. Very large local field intensities are predicted around the band edge wavelengths, in particular for the upper band edge. Nonlinear propagation is studied through the analysis of degenerate four-wave mixing. For counterpropagating pump beams orthogonally sent onto the 2D-PC huge improvement of the phase conjugate reflectivity are predicted at least for small incidence angles of the signal beam. These results represent an improvement by a factor of 20 when compared to the case of a 1D-PC of the same thickness made of the same materials. As three intense phase conjugate beams are generated in the four-wave mixing interaction, the 2D-PC could be very interesting for the purpose of dense parallel optical signal processing. Moreover, the simple theoretical analysis developed in the paper can be used for any kind of 2D-PC.
\end{abstract}




\section{Introduction}

Since the discovery of photonic crystals (PC) $[1,2]$ a lot of theoretical and experimental works have been performed so that practical devices for optical microcircuits may now be considered for optical signal processing [3,4]. However, such microcircuits require optical nonlinear functions such as those provided by third order nonlinear processes. Unfortunately the best nonlinear materials such as semiconductors exhibit too small optical nonlinearities which prevent their use at low power in small thickness devices. However, light localization properties of photonic crystals linked to the group velocity reduction near a band extremum enables a large increase of light intensity inside the structure which is particularly interesting for nonlinear effects [5-11]. The strong enhancement of the nonlinear optical susceptibility was quantified using the local field factor [12]. The simplest case of 1D-PC was first studied and large local field factors leading to large increases in the efficiency of nonlinear processes predicted [13] and measured $[14,15]$ in such PC's.

At this point one question can be asked: is a 2D-PC more efficient than a 1Done for enhancing optical nonlinearities? At first sight, since there is less nonlinear material in a conventional air-semiconductor $2 \mathrm{D}-\mathrm{PC}$ than in a $1 \mathrm{D}-\mathrm{PC}$, the only possibility for a better nonlinear efficiency of the $2 \mathrm{D}-\mathrm{PC}$ lies in the potentiality of getting a higher local field in an optimized 2D-PC. Due to the hardness of fabrication of such PC's a preliminary theoretical study is evidently wished. Several methods have been used to solve nonlinear propagation problems in 2D-PC's: the finite-difference time-domain (FDTD) often used to calculate band displacements due to Kerr effect [16-19], the Fourier factorisation of nonlinear Maxwell equations with application to Kerr effect [20, 21], the variational method for the description of optical solitonic waves [22] or tunable super prism effect [23], the Bloch mode method [24-26] or the effective field methods summing 
over several Bloch modes of about the same wavevector [27, 28]. All these methods provide good numerical results when applied to particular 2D-PC, but due to their complexity, it is difficult to derive general statements for optimizing the pattern of the 2DPC.

In this article, we propose a particular 2D-PC which is potentially interesting since it benefit from a degeneracy of the band edge wavelength for different diffracted beams. For the theoretical analysis of this $2 \mathrm{D}-\mathrm{PC}$ we use a simple method based on coupled-waves for the calculation of the local field and the degenerate four-wave mixing near this degenerate band edge wavelength.

Section II describes the 2D-PC considered in this study for the degeneracy of its band edge and provides the Fourier expansion of its linear and nonlinear optical susceptibilities. Section III considers the linear propagation in the 2D-PC in the particular case when the wavelength of the incident beam is located in the vicinity of the PC band edge. The simple calculations used in our approach are presented and results are discussed concerning the value of the local intensity inside the 2D-PC. The study is enlarged to nonlinear propagation in section IV in the case of degenerate four-wave mixing. In this section calculation of the conjugate reflectivity for the considered configuration of the different beams is developed and results analyzed in comparison with those obtained for a 1D-PC of the same thickness. Finally, Appendix I is devoted to the justification of the coupled-wave approximation used all along our analysis.

\section{The 2D Photonic Crystal}

In order to benefit from the degenerate band edge we consider in this paper the 2D-PC shown in figure 1.A. This 2D-PC is infinite in the $x$ direction and has a thickness $\mathrm{L}$ in the $z$ direction. Such a photonic crystal can be obtained by etching of a material of refractive index $n_{1}$ with the etched part filled by a material of refractive index $n_{2}$. This PC results 
from the etching of two tilted gratings of the same period $\Lambda=e_{1}+e_{2}$ and the same filling factor $e_{1} / \Lambda$ with wavevectors $\underline{K_{1}}$ and $\underline{K_{2}}$ tilted by angles $+\alpha$ and $-\alpha$ with respect to the $z$ direction respectively. These gratings are shown in dotted lines in figure 1.A. The 2D-PC is therefore composed of diamond-shaped pins of refractive index $n_{1}$ located in a sea of refractive index $n_{2}$ arranged following a centred rectangular lattice. The primitive lattice cell shown in figure 1.B is a rectangle with dimensions $\Lambda / \cos (\alpha)$ and $\Lambda / \sin (\alpha)$ along the $z$ and $x$ directions respectively. The diamond of diagonals $e_{1} / \cos (\alpha)$ and $e_{1} / \sin (\alpha)$ located at the centre of the cell and the triangles of sides $e_{1} /(2 \cos (\alpha))$ and $e_{1} /(2 \sin (\alpha))$ occupying its four corners have the refractive index $n_{1}$, the remaining part of the unit cell exhibiting a refractive index $n_{2}$. Taking the periodicity of the 2D-PC into account the linear permittivity $\varepsilon(z, x)$ and the $\mathrm{n}^{\text {th }}$ order nonlinear susceptibility $\chi^{(n)}(z, x)$ can be decomposed using Fourier series following the relation

$$
\xi(z, x)=\left[\sum_{N_{z}, N_{x}} \xi_{N_{z}, N_{x}} \exp i\left[N_{z} K_{z} z+N_{x} K_{x} x\right]\right] \Pi\left(\frac{z}{L}\right)
$$

With $\xi=\varepsilon$ or $\chi^{(n)}$. In equation II. $1 N_{z}$ and $N_{x}$ are positive, negative, or null integers, $K_{z}=2 \pi \cos (\alpha) / \Lambda$ and $K_{x}=2 \pi \sin (\alpha) / \Lambda$ are the modulus unit cell wavevectors in the reciprocal space, and $\Pi(z / L)=1$ for $0 \leq z \leq L$ and $\Pi(z / L)=0$ elsewhere. The $\left(N_{z}, N_{x}\right)$ order Fourier component $\xi_{N_{z} N_{x}}$ given by

$$
\xi_{N_{z} N_{x}}=\int_{-\Lambda / 2 \cos \alpha}^{+\Lambda / 2 \cos \alpha} d z \int_{-\Lambda / 2 \sin \alpha}^{+\Lambda / 2 \sin \alpha} d x \xi(z, x) \exp i\left(N_{z} K_{z} z+N_{x} K_{x} x\right)
$$

takes the value

$$
\xi_{0,0}=\xi_{2}+\left(\xi_{1}-\xi_{2}\right) \frac{e_{1}^{2}}{\Lambda^{2}}
$$




$$
\xi_{N_{z}, N_{x}}=\frac{2\left(\xi_{1}-\xi_{2}\right)}{\pi^{2}\left(N_{z}^{2}-N_{x}^{2}\right)} \sin \left[\frac{\pi}{2}\left(N_{z}+N_{x}\right) \frac{e_{1}}{\Lambda}\right] \sin \left[\frac{\pi}{2}\left(N_{z}-N_{x}\right) \frac{e_{1}}{\Lambda}\right]\left\{1+\exp \left[-i \pi\left(N_{z}-N_{x}\right)\right]\right\}
$$

where $\xi_{1}$ and $\xi_{2}$ are the values of $\xi(z, x)$ in the materials of refractive indices $n_{1}$ and $n_{2}$ respectively. As expected from the symmetry of the unit cell, $\xi_{N_{z},-N_{x}}=\xi_{-N_{z},-N_{x}}=\xi_{-N_{z}, N_{x}}=\xi_{N_{z}, N_{x}}$. Note also that $\xi_{N_{z}, N_{x}}=0$ if $N_{z}-N_{x}$ is odd and that $\xi_{N_{z}, N_{x}}$ reduces to

$$
\xi_{N_{z}, \pm N_{z}}=\frac{\xi_{1}-\xi_{2}}{\pi N_{z}} \frac{e_{1}}{\Lambda} \sin \left[\pi N_{z} \frac{e_{1}}{\Lambda}\right]
$$

for $N_{x}= \pm N_{z}$.

Let as note also that, in order to separate band edge effects which are our prime interest in the present analysis from Fabry-Perot resonances, the 2D-PC is assumed to be surrounded by a medium of refractive index $n_{0}=\sqrt{\varepsilon_{00}}$, or, equivalently anti-reflexion coated or slightly prismatic. In such a way there is no unwanted reflexion at the boundaries which might artificially enhance the field intensities.

\section{Linear propagation and local intensity}

For the sake of simplicity we consider here isotropic diffraction of an incident beam $\underline{E_{R}}(z, x)$ polarized orthogonally to the incidence plane (i.e. in the y direction) with all the possible diffracted waves also polarized along the y direction. The total electromagnetic field $\underline{E}(z, x)$ inside the sample $(0 \leq z \leq L)$ writes then:

$$
\underline{E}(z, x)=\sum_{N^{\prime}} \underline{E}_{D_{N^{\prime}}}(z, x)
$$

where, for $N^{\prime}=0, \underline{E}_{D_{0}}(z, x)=\underline{E}_{R}(z, x)=\hat{e} A_{R} \exp i \underline{k}_{R} \cdot \underline{r}$ is the incident field of polarization vector $\hat{e}$, complex amplitude $A_{R}$ and wavevector $\underline{k}_{R}=k\left(\cos \theta_{R} \hat{z}+\sin \theta_{R} \hat{x}\right)$, 
and $\underline{E}_{D_{N^{\prime}}}=\hat{e} A_{D_{N^{\prime}}} \exp \left(i \underline{k}_{D_{N^{\prime}}} \underline{r}\right)$ is the $N^{\prime}$ th order diffracted wave of complex amplitude $A_{D_{N^{\prime}}}$ and wavevector $\left.\underline{k}_{D_{N^{\prime}}}=k \mid \cos \theta_{D_{N}} \hat{z}+\sin \theta_{D_{N}} \hat{x}\right\rfloor$ (see figure 2). $k=2 \pi_{n_{0}} / \lambda$ is the modulus of each wavevector $\underline{k}_{D_{N^{\prime}}}$ with $\lambda$ the incident beam wavelength and $n_{0}=\sqrt{\varepsilon_{00}}$ the mean refractive index of the 2D-PC.

In the following the slowly varying envelop approximation (SVEA) is used for the description of beam propagation although large local field factors are predicted in section III.4. at the band edge of the short length 2D-PC considered in our analysis. In fact, this SVEA approximation is valid in a medium possessing the translational symmetry in the x-direction because, as long as, as shown here after (see equations III.6) the read and reflected Bragg waves are considered in our analysis. Indeed, these diffracted beams play the role of the counterpropagating waves existing when the SVEA is not made [29]. The validity of such an approximation at the band edge of 1D-PC's was already proven in Ref. 13. The propagation equation of the amplitude $A_{D_{N^{\prime}}}$ then writes:

$$
2 i \underline{k}_{D_{N^{\prime}}} \cdot \underline{\nabla} A_{D_{N^{\prime}}}=\sum_{N_{z} N_{x^{\prime}} \sum^{\prime \prime}} \varepsilon_{N_{z} N_{x}} A_{D_{N^{\prime \prime}}} \exp i\left[\left(\underline{k}_{D_{N^{\prime \prime}}}-\underline{k}_{D_{N}}\right) \cdot \underline{r}+N_{z} K_{z} z+N_{x} K_{x} x\right\rfloor
$$

The grating being infinite in the $\mathrm{x}$ direction, phase-matching is required along this direction, which gives:

$$
k\left[\sin \left(\theta_{D_{N^{\prime \prime}}}\right)-\sin \left(\theta_{D_{N^{\prime}}}\right)\right]+N_{x} K_{x}=0
$$

Equation III.3 means that diffracted waves of orders $N^{\prime}$ and $N$ " are coupled through the order $N_{x}$ of the 2D-grating along the $\mathrm{x}$ direction and to all the grating orders along the $\mathrm{z}$ direction. The phase mismatch is then along the $\mathrm{z}$ direction only and writes:

$$
\Delta k_{N^{\prime}, N_{z}, N_{x}} L=N_{z} K_{z} L+k\left(\cos \theta_{D_{N^{\prime \prime}}}-\cos \theta_{D_{N}}\right) L
$$

\section{III.1. Small phase-mismatch approximation at the PC band edge}


Relation III.3 also holds for $N^{\prime \prime}=0$ corresponding to the incident wave so that diffracted waves are emitted in transmission and in reflexion in directions $\theta_{D_{N^{\prime}}}=\operatorname{Arcsin}\left(\frac{N_{x} \lambda \sin (2 \alpha)}{\lambda_{0}}+\sin \theta_{R}\right)=\theta_{0} \quad$ and $\quad \theta_{D_{N^{\prime}}}=\pi-\theta_{0}$ respectively. $\quad$ In $\quad$ the following, we will restrict ourselves to the most interesting case when the diffracted orders corresponding to $N_{x}= \pm 1$ are at the degenerate band edge of the 2D-PC. Indeed, as shown in Appendix I only these waves exhibit the high local intensities required for nonlinear optics.

Two conditions are necessary for a strong coupling between incident and diffracted waves: a high Fourier coefficient $\varepsilon_{N_{z}, N_{x}}$ and a quasi-perfect phase matching $\left(\Delta k_{N^{\prime}, N_{z}, N_{x}} L \approx 0\right)$. As shown by equation II.4 the highest possible coupling occurs through the $N_{z}= \pm 1$ components $\varepsilon_{ \pm 1, \pm 1}=\varepsilon_{11}=\frac{\varepsilon_{1}-\varepsilon_{2}}{\pi} \frac{e_{1}}{\Lambda} \sin \left[\pi \frac{e_{1}}{\Lambda}\right]$ of the Fourier series describing $\varepsilon(z, x)$. We therefore consider a $2 \mathrm{D}$-PC of periods $\Lambda / \cos \alpha$ and $\Lambda / \sin \alpha$ such as the phase-mismatch $\Delta k_{0,1, \pm 1} L$ is null for $N_{z}=1$ at the centre of the degenerate forbidden band for a read beam of wavelength $\lambda_{0}$ orthogonally incident $\left(\theta_{R}=0\right)$ onto the 2D-PC. In such a situation, $\lambda_{0}, \Lambda$, and $\alpha$ are linked through the relation:

$$
\lambda_{0}=2 \Lambda n_{\text {eff }} \cos \alpha
$$

In the small phase-mismatch approximation used in our analysis, due to relation III.5, only the waves diffracted in reflexion in orders $N_{x}= \pm 1$ are considered. Other diffracted waves (in transmission and reflexion) present large phase mismatches and are safely neglected. In the same manner, only orders $N_{z}= \pm 1$ are considered in our calculations. Although they must be taken into account in particular for an exact determination of the band edge wavelength, because they exhibit large phase mismatches, they can be safely neglected 
too. The validity of this small phase-mismatch approximation is discussed in details and proved in Appendix 1.

\section{III.2. Reflectivity}

In the small phase-mismatch approximation the coupled propagation equations derived from equation III. 2 writes:

$$
\begin{array}{lrl}
\frac{d A_{R}}{d z}=\frac{i \pi \varepsilon_{11}}{n_{e f f} \lambda \cos \theta_{R}}\left[A_{D_{1}} \exp i \Delta k_{1} z+A_{D_{2}} \exp i \Delta k_{2} z\right] & \text { III.6.a } \\
\frac{d A_{D_{1}}}{d z}=\frac{i \pi \varepsilon_{11}}{n_{\text {eff }} \lambda \cos \theta_{D_{1}}} A_{D_{1}} \exp -i \Delta k_{1} z & \text { III.6.b } \\
\frac{d A_{D_{2}}}{d z}=\frac{i \pi \varepsilon_{11}}{n_{\text {eff }} \lambda \cos \theta_{D_{2}}} A_{D_{2}} \exp -i \Delta k_{2} z & \text { III.6.c }
\end{array}
$$

With $\quad \theta_{D_{1}}=\pi-A r c \sin \left\lfloor\sin \theta_{R}+\frac{\lambda \sin \alpha}{\Lambda n_{\text {eff }}}\right\rfloor \quad, \quad \theta_{D_{2}}=\pi-\operatorname{Arcsin}\left\lfloor\sin \theta_{R}-\frac{\lambda \sin \alpha}{\Lambda n_{\text {eff }}}\right\rfloor \quad, \quad$ and $\Delta k_{1,2}=\frac{2 \pi \cos \alpha}{\Lambda}+\frac{2 \pi n_{\text {eff }}}{\lambda}\left[\cos \theta_{D_{1,2}}-\cos \theta_{R}\right] . \quad$ Setting $\quad A_{R}=a_{R} \exp i\left(\frac{\Delta k_{1}+\Delta k_{2}}{4}\right) z$ $A_{D_{1}}=a_{1} \exp i\left(\frac{\Delta k_{2}-3 \Delta k_{1}}{4}\right) z, \quad$ and $\quad A_{D_{2}}=a_{2} \exp i\left(\frac{\Delta k_{1}-3 \Delta k_{2}}{4}\right) z, \quad$ and $\quad$ using $\quad$ a matrix representation equations III.6.a to $\mathrm{c}$ write

$$
\frac{d A}{d z}=M A
$$

with

$$
A=\left[\begin{array}{l}
a_{R} \\
a_{1} \\
a_{2}
\end{array}\right]
$$

And 


$$
M=\left[\begin{array}{ccc}
-i\left(\Delta k_{1}+\Delta k_{2}\right) / 4 & i \frac{\pi \varepsilon_{1}}{n_{e f f} \lambda \cos \theta_{R}} & i \frac{\pi \varepsilon_{1}}{n_{e f f} \lambda \cos \theta_{R}} \\
i \frac{\pi \varepsilon_{1}}{n_{e f f} \lambda \cos \theta_{D_{1}}} & -i\left(\Delta k_{2}-3 \Delta k_{1}\right) / 4 & 0 \\
i \frac{\pi \varepsilon_{1}}{n_{e f f} \lambda \cos \theta_{D_{2}}} & 0 & -i\left(\Delta k_{1}-3 \Delta k_{2}\right) / 4
\end{array}\right]
$$

III.8

Resolution of the matrix differential equation III.7 is performed through the diagonalization of the matrix $\mathrm{M}$ by using the basis change given by the matrix

$$
P=\left[\begin{array}{lll}
x_{1} & x_{2} & x_{3} \\
y_{1} & y_{2} & y_{3} \\
z 1 & z_{2} & z_{3}
\end{array}\right]
$$

With $\left(x_{i}, y_{i}, z_{i}\right)$ the components of the eigenvectors of the new basis. In the new basis, equation III.7 writes

$$
\frac{d B}{d z}=D B
$$

With $B=P^{-1} A$ and $D=P^{-1} M P$ the diagonal matrix representing the $\mathrm{M}$ matrix in the new basis. Integration of equation III.10 with the limit conditions $A_{R}(0)=E_{R 0}, A_{D_{1}}(L)=0$, and $A_{D_{2}}(L)=0$ together with a reciprocal basis change allows to deduce the transmission $T_{\mathrm{R}}$ and reflectivities $R_{1}$ and $R_{2}$ of the device

$$
\begin{gathered}
T_{R}=\left|E_{R}(L)\right|^{2} /\left|E_{R 0}\right|^{2}=\left|x_{1} B_{R} \exp \lambda_{1} L+x_{2} B_{1} \exp \lambda_{2} L+x_{3} B_{2} \exp \lambda_{3} L\right|^{2} /\left|E_{R 0}\right|^{2} \\
R_{1}=\left(\cos \theta_{D_{1}} / \cos \theta_{R}\right)\left|E_{D_{1}}(0)\right|^{2} /\left|E_{R 0}\right|^{2}=\left(\cos \theta_{D_{1}} / \cos \theta_{R}\right)\left|y_{1} B_{R}+y_{2} B_{1}+y_{3} B_{2}\right|^{2} /\left|E_{R 0}\right|^{2} \\
R_{2}=\left(\cos \theta_{D_{2}} / \cos \theta_{R}\right)\left|E_{D_{2}}(0)\right|^{2} /\left|E_{R 0}\right|^{2}=\left(\cos \theta_{D_{2}} / \cos \theta_{R}\right)\left|z_{1} B_{R}+z_{2} B_{1}+z_{3} B_{2}\right|^{2} /\left|E_{R 0}\right|^{2}
\end{gathered}
$$

With $B_{\mathrm{R}}, B_{1}$, and $B_{2}$ the components of the $\mathrm{B}$ vector solutions of the system of equations provided by limit conditions:

$$
\left\{\begin{array}{l}
x_{1} B_{R}+x_{2} B_{1}+x_{3} B_{2}=E_{R 0} \\
y_{1} B_{R} \exp \lambda_{1} L+y_{2} B_{1} \exp \lambda_{2} L+y_{3} B_{2} \exp \lambda_{3} L=0 \\
z_{1} B_{R} \exp \lambda_{1} L+z_{2} B_{1} \exp \lambda_{2} L+z_{3} B_{2} \exp \lambda_{3} L=0
\end{array}\right.
$$


And $\lambda_{\mathrm{i}}(\mathrm{i}=1,3)$ the diagonal elements of $D$.

In the general case the transmissions and reflectivities are numerically computed. However, in the case when the read beam is normal to the 2D-PC, the diffracted beams are symmetrical with respect to the incident beam and analytical solutions are found for $T_{\mathrm{R}}$ and $R_{1}$ and $R_{2}$ :

$$
\begin{gathered}
T=T_{R}=\left|\frac{A_{R}(L)}{E_{R 0}}\right|=\frac{4|\beta|^{2}}{|2 \beta \operatorname{ch} \beta L+i \Delta k s h \beta L|^{2}} \\
R=R_{1}=R_{2}=\cos 2 \alpha^{\prime}\left|\frac{A_{D 1,2}(0)}{E_{R 0}}\right|^{2}=2 \beta_{0}^{2}\left|\frac{\operatorname{sh} \beta L}{2 \beta \operatorname{ch} \beta L+i \Delta k s h \beta L}\right|^{2}
\end{gathered}
$$

Where $\quad \beta^{2}=\beta_{0}^{2}-\frac{\Delta k^{2}}{4}, \quad \beta_{0}^{2}=\frac{2 \pi^{2} \varepsilon_{1}^{2}}{n_{\text {eff }}^{2} \lambda^{2} \cos \alpha^{\prime}}, \quad \alpha^{\prime}=\frac{1}{2} \operatorname{Arcsin}\left(\frac{\lambda \sin \alpha}{\Lambda n_{\text {eff }}}\right)$, and

$$
\Delta k=\frac{2 \pi \cos \alpha}{\Lambda}+\frac{2 \pi n_{e f f}}{\lambda}\left(\cos \alpha^{\prime}\right)
$$

Note that, as it must be, energy is preserved since one gets $2 R+T=1$ for the analytical solution given by equations III.13.a-b as well as $R_{1}+R_{2}+T_{R}=1$ for the numerical one provided by equations III.11.a-c.

\section{III.3. Local Intensity}

The local field inside the 2D-PC is given by

$$
E(z, x)=A_{R}(z) \exp \underline{k}_{R} \cdot \underline{r}+A_{D_{1}}(z) \exp i \underline{k}_{D_{1}} \cdot \underline{r}+A_{D_{2}}(z) \exp i \underline{k}_{D_{2}} \cdot \underline{r}
$$

With

$$
\begin{aligned}
& A_{R}(z)=\left(x_{1} B_{R} \exp \lambda_{1} z+x_{2} B_{1} \exp \lambda_{2} z+x_{3} B_{2} \exp \lambda_{3} z\right) \exp i \frac{\Delta k_{1}+\Delta k_{2}}{4} z \\
& A_{D_{1}}(z)=\left(y_{1} B_{R} \exp \lambda_{1} z+y_{2} B_{1} \exp \lambda_{2} z+y_{3} B_{2} \exp \lambda_{3} z\right) \exp i \frac{\Delta k_{2}-3 \Delta k_{1}}{4} z \\
& A_{D_{2}}(z)=\left(z_{1} B_{R} \exp \lambda_{1} z+z_{2} B_{1} \exp \lambda_{2} z+z_{3} B_{2} \exp \lambda_{3} z\right) \exp i \frac{\Delta k_{1}-3 \Delta k_{2}}{4} z
\end{aligned}
$$

The local intensity is then given by $I(z, x)=|E(z, x)|^{2}$ 


\section{III.4. Numerical Results}

In this section the incident beam is sent perpendicularly onto the 2D-PC described in section II. All the calculations were performed using $\varepsilon_{1}=n_{1}^{2}=10.43, \varepsilon_{2}=n_{2}^{2}=1, \alpha=20$ degrees for a sample of length $L=4 \mu \mathrm{m}$. In order to give an insight into the potential optimization of the device for applications, the band-centre wavelength $\lambda_{0}$ was adjusted so that the upper or lower band edge wavelength $\lambda_{B}=1500 \mathrm{~nm}$.

The influence of the filling factor $e_{1} / \Lambda$ on the normalized mean local intensity $I_{L}=\left(1 /\left(I_{0} L \Lambda \sin (\alpha)\right)\right) \int_{-\Lambda / 2 / \sin (\alpha)}^{+\Lambda / 2 / \sin (\alpha)} d x \int_{0}^{L} d z I(x, z)$ was studied for both upper and lower band edges. The result is presented in figures 3.A and B together with the grating wavelength $\Lambda=\lambda_{0} /\left(2 n_{\text {eff }} \cos (\alpha)\right)$ for the cases of lower and upper bad edges respectively. In both cases, due to the increase of the effective index of refraction consecutive to the lower volume of the etched part of the 2D-PC, the grating wavelength decreases with increasing values of $e_{1} / \Lambda$. It can also be observed from figure 3 that the filling factor plays an important role in enhancing the local intensity with a maximum around $e_{1} / \Lambda=0.5$ in both cases. Figure 3 also shows that the mean local intensity is much larger on the upper band edge of the 2D-PC. This is directly connected to the larger number of periods obtained in this case for the same device length. This means that better results are expected in the nonlinear regime when the upper band edge of the 2D-PC is considered. As a consequence, only this case with an optimized filling factor $\left(e_{1} / \Lambda=0.5\right)$ is considered in the following.

Due to the symmetry of the problem, for an incident beam orthogonal to the 2D-PC both diffracted beams exhibit the same reflectivity. Figure 4.A shows the reflectivity spectrum of one of these two identical diffracted beams together with that of the normalized mean local intensity $I_{L}$. At low wavelengths, the reflectivity of each diffracted beam tends towards one half and the device is totally reflecting with two equal intensity diffracted beams. 
At the band edge occurring at $1500 \mathrm{~nm}$ the reflectivity drops to zero and the mean normalized local intensity is maximum. Note that the resonance leads to high local intensities $\left(I_{L}=34\right)$ and to narrow bandwidths $(\Delta \lambda=3.3 \mathrm{~nm})$ which can be useful for optical filtering or WDM optical signal processing.

Figure 5 shows the spatial repartition of the local intensity inside the 2D-PC. The pattern which is infinite in the $x$-direction is only represented between $x=0$ and $x=4 \mu \mathrm{m}$ in the figure. Local intensity is patterned following the 2D-PC etching with maximum at the centre of the device in the z-direction with intensity almost 200 times larger than the incident intensity which is a great promise of efficiency for nonlinear optical processes. It is also important to note that much higher local field intensities can be obtained using longer PC's, but with somewhat narrower frequency bandwidths.

\section{Degenerate Four-wave Mixing}

Four-wave mixing is used for characterizing the local field induced enhancement of the third order optical nonlinear susceptibility [12] and to compare the efficiency of 2D-PC for this purpose to the case of already known 1D-PC [13].

\section{IV.1. Optical configuration}

The optical configuration considered in this section is shown in figure 6.A and B. Figure 6.A represents the beams incident onto the $2 \mathrm{D}-\mathrm{PC}$ and the beams that outcomes from this $\mathrm{PC}$, while the wavevectors of the different waves present in the sample are indicated in figure 6.B.

The two counter propagating incident pump wave $\underline{E_{R F}}(0)$ and $\underline{E_{R B}}(L)$ are orthogonal to the 2D-PC and give rise to six pump waves inside the PC. These six waves are counter propagating two by two: the forward and backward reference waves $\left(\underline{E_{R F}}\right.$ and $\left.\underline{E_{R B}}\right)$ due to the transmission of the incident pump waves and the waves $\left(\underline{E}_{D F_{1}}\right.$ and $\underline{E_{D B_{1}}}$, and 
$\underline{E_{D F_{2}}}$ and $E_{D B_{2}}$ ) diffracted by the reference waves (see the corresponding wavevectors in figure 6.B). The incident signal beam $\underline{E_{S}}(0)$ gives rise to three signal waves $\left(\underline{E_{S}}, \underline{E_{S D_{1}}}\right.$, and $\left.\underline{E_{S D_{2}}}\right)$ and three conjugate waves $\left(\underline{E_{C}}, \underline{E_{C D_{1}}}\right.$, and $\left.\underline{E_{C D_{2}}}\right)$ counter propagating to $\left(\underline{E_{S}}, \underline{E_{S D_{1}}}\right.$, and $E_{S D_{2}}$ ) respectively.

In the following, calculations performed for the determination of the propagation of the different waves are presented before giving some numerical results and comparing these results with those obtained when using 1D-PC. All the calculations were performed in the fully parametric approximation of no significant pump depletion and signal amplification. Note that this approximation is always possible when considering the fourwave mixing process provided the pump and signal incident intensities are low enough. This approximation also allows describing the pump and signal propagation in the linear regime in order to show the local field enhancement of the third order nonlinear susceptibilities with no spurious effects. At higher pump intensities Kerr effect induced self phase modulation and cross phase modulation are evidently expected. These processes could also be treated using the formalism described in this paper.

\section{IV.2. Pump wave propagation}

The amplitudes of the pump waves $\underline{E_{R F}}, \underline{E_{D F_{1}}}$, and $\underline{E_{D F_{2}}}$ issued from the forward propagating incident pump beam are directly derived from results of section III.2 with $\theta_{R F}=\theta_{R}=0$. The amplitudes of the pump waves $\underline{E_{R B}}, \underline{E_{D B_{1}}}$, and $\underline{E_{D B_{2}}}$ issued from the forward propagating incident pump beam are also derived from results of section III.2, but with $\theta_{R B}=\pi$, replacing $K_{Z}$ by $-K_{Z}$, and using adequate limit conditions $\left(\underline{E}_{R B}(L)=E_{R B_{0}}, \underline{E_{D B_{1}}}(0)=0, \underline{E}_{D B_{2}}(0)=0\right)$. One gets then: 


$$
\begin{aligned}
& A_{R I}^{(j)}(z)=\left[x_{1}(j) B_{R}(j) \exp \left(\lambda_{1}(j) z\right)+x_{2}(j) B_{1}(j) \exp \left(\lambda_{2}(j) z\right)\right. \\
& \left.+x_{3}(j) B_{2}(j) \exp \left(\lambda_{3}(j) z\right)\right] \exp i\left(\frac{\Delta k_{R I_{1}}(j)+\Delta k_{R I_{2}}(j)}{4}\right) z \\
& A_{D I_{1}}^{(j)}(z)=\left[y_{1}(j) B_{R}(j) \exp \left(\lambda_{1}(j) z\right)+y_{2}(j) B_{1}(j) \exp \left(\lambda_{2}(j) z\right)\right. \\
& \left.+y_{3}(j) B_{2}(j) \exp \left(\lambda_{3}(j) z\right)\right] \exp i\left(\frac{\Delta k_{R I_{2}}(j)-3 \Delta k_{R I_{1}}(j)}{4}\right) z \\
& A_{D I_{2}}^{(j)}(z)=\left[z_{1}(j) B_{R}(j) \exp \left(\lambda_{1}(j) z\right)+z_{2}(j) B_{1}(j) \exp \left(\lambda_{2}(j) z\right)\right. \\
& \left.+z_{3}(j) B_{2}(j) \exp \left(\lambda_{3}(j) z\right)\right] \exp i\left(\frac{\Delta k_{R I_{1}}(j)-3 \Delta k_{R I_{2}}(j)}{4}\right) z \quad \text { IV.1.c } \\
& (I \equiv F, B ; j=0, \pi)
\end{aligned}
$$

With $\Delta k_{R I_{1,2}}=\frac{2 \pi \cos \alpha}{\Lambda}+\frac{2 \pi n_{e f f}}{\lambda}\left[\cos \theta_{D I_{1,2}}-\cos \theta_{R I}\right],(I \equiv F, B)$

\section{IV.3. Signal wave propagation}

Signal waves $\underline{E_{S}}, \underline{E_{S D_{1}}}$, and $\underline{E_{S D_{2}}}$ are also given by equations IV.1.a-c if using $j=\theta_{S}$ and $R I \equiv S$ and $D I \equiv S D$

\section{IV.4. Conjugate wave propagation}

Conjugate waves $\underline{E_{C}}, \underline{E_{C D_{1}}}$, and $\underline{E_{C D_{2}}}$ are coupled to each other by the 2D-PC and to signal waves $\underline{E_{S}}, \underline{E_{S D_{1}}}$, and $\underline{E_{S D_{2}}}$ through four-wave mixing with the pump waves $\underline{E_{R F}}, \underline{E_{D F_{1}}}$, $\underline{E_{D F_{2}}}, \underline{E_{R B}}, \underline{E_{D B_{1}}}$, and $\underline{E_{D B_{2}}}$. Since the conjugate waves are counter-propagating to the signal waves, the propagation equations of their amplitudes $A_{C}, A_{C D_{1}}$, and $A_{C D_{2}}$ are then given by:

$$
\begin{aligned}
\frac{d A_{C}}{d z}=-\frac{i \pi}{n_{e f f} \lambda \cos \theta_{S}} & \varepsilon_{1}\left[A_{C D_{1}} \exp -i \Delta k_{S 1} z+A_{C D_{2}} \exp -i \Delta k_{S 2} z\right] \\
& -\frac{i 4 \pi^{2}}{n_{\text {eff }} \lambda \cos \theta_{S}} \chi_{\text {eff }}^{(3)}\left[A_{R F} A_{R B}+A_{D F_{1}} A_{D B_{1}}+A_{D F_{2}} A_{D B_{2}}\right] A_{S}^{*}
\end{aligned}
$$




$$
\begin{aligned}
& \frac{d A_{C D_{1}}}{d z}=-\frac{i \pi}{n_{e f f} \lambda \cos \theta_{S D_{1}}} \varepsilon_{1}\left[A_{C} \exp i \Delta k_{S 1} z\right] \\
& \quad-\frac{i 4 \pi^{2}}{n_{e f f} \lambda \cos \theta_{S_{D 1}}} \chi_{e f f}^{(3)}\left[A_{R F} A_{R B}+A_{D F_{1}} A_{D B_{1}}+A_{D F_{2}} A_{D B_{2}}\right] A_{S D_{1}}^{*} \\
& \frac{d A_{C D_{2}}}{d z}=-\frac{i \pi}{n_{e f f} \lambda \cos \theta_{S D_{2}}} \varepsilon_{1}\left[A_{C} \exp i \Delta k_{S 2} z\right] \\
& -\frac{i 4 \pi^{2}}{n_{e f f} \lambda \cos \theta_{S D_{2}}} \chi_{e f f}^{(3)}\left[A_{R F} A_{R B}+A_{D F_{1}} A_{D B_{1}}+A_{D F_{2}} A_{D B_{2}}\right] A_{S D_{2}}^{*}
\end{aligned}
$$

Where $\chi_{\text {eff }}^{(3)}=\chi_{0,0}^{(3)}$ as given by equation II.3.a. The first term of the right hand side of equations IV.2.a-c corresponds to Bragg diffraction (as in section III.6) and the second one to the four-wave mixing process. Setting $A_{S}=a_{S} \exp i\left(\frac{\Delta k_{S 1}+\Delta k_{S 2}}{4}\right) z$,

$$
\begin{array}{lr}
A_{C}=a_{C} \exp -i\left(\frac{\Delta k_{S 1}+\Delta k_{S 2}}{4}\right) z, & A_{S_{D 1}}=a_{S_{D 1}} \exp i\left(\frac{\Delta k_{S 2}-3 \Delta k_{S 1}}{4}\right) z, \\
A_{C_{D 1}}=a_{C_{D 1}} \exp -i\left(\frac{\Delta k_{S 2}-3 \Delta k_{S 1}}{4}\right) z, & A_{S_{D 2}}=a_{S_{D 2}} \exp i\left(\frac{\Delta k_{S 1}-3 \Delta k_{S 2}}{4}\right) z,
\end{array}
$$
$A_{C_{D 2}}=a_{C_{D 2}} \exp i\left(\frac{\Delta k_{S 1}-3 \Delta k_{S 2}}{4}\right) z$, equations IV.2.a-c can be written in matrix form:

$$
\frac{d}{d z} A_{C}=M_{C} A_{C}+A_{C}^{\prime}
$$

With $A_{C}=\left(\begin{array}{c}a_{C} \\ a_{C D_{1}} \\ a_{C D_{2}}\end{array}\right)$ and $A_{C}^{\prime}=\left(\begin{array}{c}F_{C}^{\prime} \\ F_{C D_{1}}^{\prime} \\ F_{C D_{2}}^{\prime}\end{array}\right)$ where

$$
F_{C}^{\prime}(z)=-\frac{i 4 \pi^{2} \chi_{e f f}^{(3)}}{n_{e f f} \lambda \cos \theta_{S}}\left[A_{R F}(z) A_{R B}(z)+A_{D F_{1}}(z) A_{D B_{1}}(z)+A_{D F_{2}}(z) A_{D B_{2}}(z)\right] a_{S}^{*}(z)
$$

$$
F_{C D_{1}}^{\prime}(z)=-\frac{i 4 \pi^{2} \chi_{e f f}^{(3)}}{n_{\text {eff }} \lambda \cos \theta_{S D_{1}}}\left[A_{R F}(z) A_{R B}(z)+A_{D F_{1}}(z) A_{D B_{1}}(z)+A_{D F_{2}}(z) A_{D B_{2}}(z)\right] a_{S D_{1}}^{*}(z) \quad \text { IV.4.b }
$$

$$
F_{C D_{2}}^{\prime}(z)=-\frac{i 4 \pi^{2} \chi_{e f f}^{(3)}}{n_{e f f} \lambda \cos \theta_{S D_{2}}}\left[A_{R F}(z) A_{R B}(z)+A_{D F_{1}}(z) A_{D B_{1}}(z)+A_{D F_{2}}(z) A_{D B_{2}}(z)\right] a_{S D_{2}}^{*}(z) \quad \text { IV.4.c }
$$


In equation IV.3, the matrix $M_{C}$ writes

$$
M_{C}=\left(\begin{array}{ccc}
\frac{i}{4}\left(\Delta k_{S 1}+\Delta k_{S 2}\right) & -\frac{i \pi \varepsilon_{1}}{n_{e f f} \lambda \cos \theta_{S}} & -\frac{i \pi \varepsilon_{1}}{n_{\text {eff }} \lambda \cos \theta_{S}} \\
-\frac{i \pi \varepsilon_{1}}{n_{\text {eff }} \lambda \cos \theta_{S_{D 1}}} & \frac{i}{4}\left(\Delta k_{S 2}-3 \Delta k_{S 1}\right) & 0 \\
-\frac{i \pi \varepsilon_{1}}{n_{\text {eff }} \lambda \cos \theta_{S_{D 2}}} & 0 & \frac{i}{4}\left(\Delta k_{S 1}-3 \Delta k_{S 2}\right)
\end{array}\right)
$$

As in the case of the linear propagation of pump and signal waves, resolution of the matrix differential equation IV.3 is performed through the diagonalization of the matrix $M_{C}$ by using the basis change given by the matrix

$$
P_{C}=\left\lfloor\begin{array}{lll}
x_{C 1} & x_{C 2} & x_{C 3} \\
y_{C 1} & y_{C 2} & y_{C 3} \\
z_{C 1} & z_{C 2} & z_{C 3}
\end{array}\right\rfloor
$$

With $x_{C i}, y_{C i}, z_{C i}(i=1,2,3)$ the components of the eigenvectors of the new basis.

The conjugate intensities write then:

$$
\begin{gathered}
I_{C}(0)=\left|x_{C 1} b_{C}(0)+x_{C 2} b_{C D_{1}}(0)+x_{C 3} b_{C D 2}(0)\right|^{2} \\
I_{C D_{1}}(L)=\left|y_{C 1} b_{C}(L)+y_{C 2} b_{C D_{1}}(L)+y_{C 3} b_{C D_{2}}(L)\right|^{2}\left|\frac{\cos \theta_{S D_{1}}}{\cos \theta_{S}}\right| \\
I_{C D_{2}}(L)=\left|z_{C 1} b_{C}(L)+z_{C 2} b_{C D_{1}}(L)+z_{C 3} b_{C D_{2}}(L)\right|^{2}\left|\frac{\cos \theta_{S D_{2}}}{\cos \theta_{S}}\right|
\end{gathered}
$$

With

$$
\begin{aligned}
& b_{C}(z)=\left\lfloor K_{C}(0)+\int_{0}^{z} F_{C}\left(z^{\prime}\right) \exp \left(-\lambda_{C 1} z^{\prime}\right) d z^{\prime}\right\rfloor \exp \lambda_{C 1} z \\
& b_{C D_{1}}(z)=\left\lfloor K_{C D_{1}}(0)+\int_{0}^{z} F_{C D_{1}}\left(z^{\prime}\right) \exp \left(-\lambda_{C 2} z^{\prime}\right) d z^{\prime}\right\rfloor \exp \lambda_{C 2} z \\
& b_{C D_{2}}(z)=\left\lfloor K_{C D_{2}}(0)+\int_{0}^{z} F_{C D_{2}}\left(z^{\prime}\right) \exp \left(-\lambda_{C 3} z^{\prime}\right) d z^{\prime}\right\rfloor \exp \lambda_{C 3} z
\end{aligned}
$$

Where $\lambda_{C i}(i=1,2,3)$ are the eigenvalues of matrix $M_{C}$ and where 


$$
\left(\begin{array}{l}
F_{C}(z) \\
F_{C D_{1}}(z) \\
F_{C D_{2}}(z)
\end{array}\right)=P_{C}^{-1}\left(\begin{array}{l}
F_{C}^{\prime}(z) \\
F_{C D_{1}}^{\prime}(z) \\
F_{C D_{2}}^{\prime}(z)
\end{array}\right)
$$

The constant matrix $K=\left(\begin{array}{l}K_{C}(0) \\ K_{C D_{1}}(0) \\ K_{C D_{2}}(0)\end{array}\right)$ is determined by using the limit conditions $a_{C}(L)=0$, $a_{C D_{1}}(0)=0$ et $a_{C D_{2}}(0)=0$.

\section{IV.5. Numerical result}

All the results presented in this section were calculated for a maximum local intensity for the pump beam, i-e, for wavelengths lying in the vicinity of the upper band edge of the forbidden gap and using a filling factor $e_{1} / \Lambda=0.5$ and a grating period $\Lambda=319$ nm. Figures 7 .A-D show the conjugate reflectivity spectra $\rho_{C}=\left|I_{C}(0)\right|^{2} /\left|I_{S}(0)\right|^{2}, \quad \rho_{C D_{1}}=\left|I_{C D_{1}}(0)\right|^{2} /\left|I_{S}(0)\right|^{2}$, and $\rho_{C D_{2}}=\left|I_{C D_{2}}(0)\right|^{2} /\left|I_{S}(0)\right|^{2}$ plotted in semi-logarithmic scale for incidence angles of the signal beam of $0,1,2$, and 3 degrees respectively. As expected, high conjugate reflectivity peaks are predicted at the band edge of the 2D-PC. The large decrease of the conjugate reflectivities (several orders of magnitude) observed for small detunings from the band edge wavelength confirms the huge enhancement of local nonlinear susceptibilities at the band edge of PCs. Due to symmetry the conjugate reflectivities of the diffracted signal beams are identical when the signal beam is perpendicular to the $2 \mathrm{D}-\mathrm{PC}$, which is not the case for signal incidence angles of 1, 2, and 3 degrees. It can also be noted from figures 7.A-D that each of the diffracted conjugate beams exhibits a reflectivity which is about half of the direct conjugate beam, as expected from the splitting of the incident signal beam in two equal diffracted beams inside the 2D-PC.

It should be noticed that, at the band edge wavelength, there are conjugate outputs of the diffracted beams of the signal beam although there is none of these diffracted beams. 
Indeed, destructive interferences occur for these diffracted beams due to the presence of the signal input beam. This is not the case for the conjugate beams which are generated inside the 2D-PC so that diffracted conjugate beams are emitted with about the same intensity as that of the signal conjugate beam. This behavior is quite similar to that occurring in a symmetrical laser cavity where only the amplified transmitted beam exists when such a cavity is operated below threshold as an intracavity amplifier while two outputs of equal intensities are observed in the laser operation of this symmetrical cavity.

Figures 7.A-D also demonstrate the high spectral and spatial selectivity of the 2D-PC, with decreases by more than one order of magnitude for detunings of 2 nanometers or 2 degrees. This property is very encouraging for the application of such devices to multiplexed optical signal processing.

\section{IV.6. Comparison with a 1D-PC}

The huge enhancement of nonlinear processes reported in this paper for 2D-PC's using a Fourier technique was also underlined previously for 1D-PC's using the transfer matrix method [13]. In the following a comparison of the performances of 1D- and 2D-PC's is described. For this comparison the same global device is considered. The two PC's are etched in a semiconductor material (dielectric permitivities $\varepsilon_{1}=n_{1}^{2}=10.43$ and $\varepsilon_{2}=n_{2}^{2}=1$ ) of length $L=4 \mu \mathrm{m}$; the band centre wavelength $\lambda_{0}$ is adjusted so that the upper band edge wavelength is $\lambda_{B}=1500 \mathrm{~nm}$, and the filling factor is adjusted for maximum mean local intensity inside the PC's. Moreover, in both cases the signal beam is orthogonal to the device. Figure 8 shows the conjugate reflectivity of the signal beam for the 1D- and the 2D-PC (dashed and continuous lines respectively). Note that the diffracted conjugate beams existing in the 2D-PC are not considered in figure 8 . The wavelength selectivity of the device is almost the same for the two PC's with a sharp resonance peak of conjugate reflectivity around the band edge at 1500nm. However, the conjugate reflectivity of the signal beam is about 20 times larger for the 2D-PC 
than that predicted for the 1D-PC. This large enhancement definitely demonstrates the favourable influence of the degeneracy of the band edge of the 2D-PC on the efficiency of nonlinear processes. Note that the global enhancement is even three times higher when considering the sum of the three conjugate beams emitted in the case of the 2D-PC. These considerations clearly means that the $2 \mathrm{D}$ configuration of the $\mathrm{CP}$ must be chosen carefully in order to maximize the nonlinear effect, which can be easily performed by using the theoretical approach described here.

\section{Conclusion}

A simple model based on the Bragg coupled equations has been developed for the description of the linear and nonlinear propagation of light in 2D-PC's. This model has been successfully applied to the particular case of a specific 2D-PC designed for exhibiting a degeneracy of its band edge in the direction of the two diffracted beams. The validity of the simple analytical model developed was demonstrated in this particular case. As the method used for the verification is quite general, it must be stressed that the model may be applied to any 2D-PC, which makes the analysis developed in this paper quite general.

The specific choice made for the 2D-PC has led to very high mean local intensities (particularly for the upper band edge of the 2D-PC) and to huge improvements of the phase conjugate reflectivity resulting from the degenerate four-wave mixing process considered for the nonlinear interaction. The advantage of this $2 \mathrm{D}$-structuration has been underlined by the comparison of the 2D-PC to a standard 1D-PC with three phase conjugate beams of individual intensity about 20 times larger than that of the single phase conjugate beam obtained with a 1D-PC. Evidently, such a property could be very interesting for high efficiency operation of high fan-out optical circuits for optical signal processing. 


\section{Appendix I: Validity of the coupled wave approximation}

In the coupled wave approximation used in section III, three waves are considered: the read wave of complex amplitude $A_{R}$ and the two Bragg diffracted waves of complex amplitudes $A_{D_{1}}$ and $A_{D_{2}}$. The diffracted wave $\underline{E_{D_{N}}}$ of wavevector $\underline{k_{D_{N}}}$ is then coupled to these three waves by the propagation equation

$$
\begin{aligned}
\frac{d A_{D_{N_{x}}}^{(j)}}{d z}=\frac{i \pi}{n_{e f f} \lambda \cos \theta_{D_{N_{x}}}^{(j)}} \sum_{N_{z} \neq 0}\left\{\varepsilon_{N_{z} ; N_{x}} A_{R} \exp -i \Delta k_{R}^{(j)}\left(N_{z}, N_{x}\right) z\right. \\
+\varepsilon_{N_{z} ;-N_{x}+1} A_{D_{1}} \exp -i \Delta k_{D_{1}}^{(j)}\left(N_{z}, N_{x}\right) z \\
\left.+\varepsilon_{N_{z} ;-N_{x}-1} A_{D_{2}} \exp -i \Delta k_{D_{2}}^{(j)}\left(N_{z}, N_{x}\right) z\right\} \quad(j=R f, T r)
\end{aligned}
$$

With

$$
\begin{gathered}
\Delta k_{R}^{(j)}\left(N_{z}, N_{x}\right)=k\left(\cos \theta_{D_{N_{x}}}^{(j)}-\cos \theta_{R}\right)+N_{z} K_{z} \\
\Delta k_{D_{1}}^{(j)}\left(N_{z}, N_{x}\right)=k\left(\cos \theta_{D_{N_{x}}}^{(j)}-\cos \theta_{D_{1}}\right)+N_{z} K_{z} \\
\Delta k_{D_{2}}^{(j)}\left(N_{z}, N_{x}\right)=k\left(\cos \theta_{D_{N_{x}}}^{(j)}-\cos \theta_{D_{2}}\right)+N_{z} K_{z}
\end{gathered}
$$

And

$$
\begin{aligned}
& \theta_{D_{-1}}^{(R)}=\theta_{D_{1}}=\pi-A r c \sin \left\lfloor\sin \theta_{R}+\frac{\lambda \sin \alpha}{\Lambda n_{\text {eff }}}\right\rfloor \\
& \theta_{D_{1}}^{(R)}=\theta_{D_{2}}=\pi-A r c \sin \left\lfloor\sin \theta_{R}-\frac{\lambda \sin \alpha}{\Lambda n_{\text {eff }}}\right\rfloor
\end{aligned}
$$

Equation A.1 considers both the transmitted waves $A_{D_{N_{x}}}^{(T r)}$ and the reflected ones $A_{D_{N_{x}}}^{(R f)}$ for $N_{x} \neq \pm 1$ where the incidence angles are given by

$$
\begin{aligned}
& \theta_{D_{N_{x}}}^{(T r)}=\operatorname{Arcsin}\left[\sin \theta_{R}-N_{x} \frac{\lambda \sin \alpha}{\Lambda n_{\text {eff }}}\right] \\
& \theta_{D_{N_{x}}^{(R f)}}=\pi-\operatorname{Arcsin}\left[\sin \theta_{R}-N_{x} \frac{\lambda \sin \alpha}{\Lambda n_{e f f}}\right]
\end{aligned}
$$


As it was made in section III only the wavelength zone lying around the upper band edge was considered here since it provides the best performances for the device. Moreover, as in section III, we considered the case of an incident beam sent quasi-perpendicularly to the 2D-PC $\left(\sin \left(\theta_{R}\right) \approx 0\right)$. For $\lambda=1.5 \mu \mathrm{m}, \lambda \sin \alpha / \Lambda / n_{\text {eff }}=0.871$ and only orders $N_{x}= \pm 1$ (in reflexion and in transmission) are possible. As a consequence, only the amplitudes $A_{D-1}^{(T r)}$ and $A_{D_{+1}}^{(T r)}$ of the transmitted diffracted waves are considered. Moreover, as $e_{1} / \Lambda=0.5$ for a maximum local intensity in the PC the non-zero Fourier components are given by the simpler equation II.4. Equation A.1 was numerically integrated for both $A_{D-1}^{(T r)}$ and $A_{D+1}^{(T r)}$ when limiting the expansion in $N_{Z}$ to the third order $\left(\left|N_{Z}\right| \leq 3\right)$. The correction due to the fifth order $\left(N_{Z}= \pm 5\right)$ was also calculated using equation A.1. The validity of the coupled-wave approximation was tested by the calculation of the local intensities $I_{L D \pm 1}^{(T r)}=\left(1 / I_{0} L\right) \int_{0}^{L}\left|A_{D \pm 1}^{T r}(z)\right|^{2} d z$ normalized to the local intensity given $I_{L}$ calculated in section III.

Figure 9 shows spectra of the third and fifth order corrections to the total local intensity due to the transmitted $N_{x}= \pm 1$ diffraction orders. It must be underlined that the error is very low (less than $1 \%$ ) in the vicinity of the band edge wavelength where the local intensity is maximum in the 2D-PC. This definitely proves the reliability of the approximation made in section III. This result also strongly confirms that the simple analysis developed in this article can be successfully applied to any nonlinear 2D-PC $(0.7 \%$ and $0.1 \%$ for the third and fifth orders corrections respectively at the band edge wavelength). This means that as far as nonlinear properties of the 2D-PC at its band edge is concerned, only the incident and Bragg diffracted waves must be considered, which definitely simplifies the analysis which can be even analytical, leakage waves due to higher order diffracted waves being calculated numerically in the linear regime. 
References

1. E. Yablonovitch, Phys. Rev. Lett., 58, 2059 (1987)

2. S. John, Phys. Rev. Lett., 58, 2486 (1987)

3. J.D. Joannopoulos, R.D. Meade, J.N. Winn (1995) Photonic crystals. Princetown University Press, New Jersey

4. T.F. Krauss, R.M. De La Rue, Progress in Quantum Electronics 23, 51 (1999).

5. W. Chen, D.L. Mills, Phys. Rev. B 36, 6269 (1987).

6. Y. Dumeige, I. Sagnes, P. Monnier, P. Vidakovic, C. Meriadec, A. Levenson, J. Opt. Soc. Am. B 19, 2094 (2002)

7. A. V. Andreev, A. V. Balakin, A. B. Kozlov, I. A. Ozheredov, I. R. Prudnikov, A. P. Shkurinov, P. Masselin, G. Mouret, J. Opt. Soc. Am. B 19, 1865 (2002)

8. G.J. Schneider, G.H. Watson, Appl. Phys. Lett. 83, 5350 (2003).

9. A. D. Bristow, J.-P. R. Wells, W. H. Fan, A. M. Fox, M. S. Skolnick, D. M. Whittaker, A. Tahraoui and T. F. Krauss, J. S. Roberts, Appl. Phys. Lett. 83, 851 (2003).

10. P. Xie, Z.Q. Zhang, Phys. Rev. A 69, 053806 (2004)

11. M. Scalora, J.P. Dowling, C.M. Bowden, M.J. Bloemer, Phys. Rev. Lett. 73, 1368 (1994)

12. R. Frey, P. Delaye, and G. Roosen. "Nonlinéarités optiques du troisième ordre dans les cristaux photoniques". Nanophotonique, eds. C. Delalande, A. Levenson, H. Rigneault, Hermès Sciences, Paris, p. 207-222 (2005) or « Third order optical nonlinearities in photonic crystals », Nanophotonics, Eds. H. Rigneault , J.M. Lourtioz, C. Delalande, A. Levenson, ISTE, p192-205 (2006).

13. P. Delaye, M. Astic, R. Frey, and G. Roosen, J. Opt. Soc. Am. B 22, 2494 (2005) 
14. L. Razzari, D. Trager, M. Astic, P. Delaye, R. Frey, G. Roosen, R. André, Appl. Phys. Lett. 86, 231106 (2005)

15. M. Astic, P. Delaye, R. Frey, G. Roosen, R. André, N. Belabas, I. Sagnes, and R. Raj, J. of Phys. D: Appl. Phys. 41, 224005 (2008)

16. P. Tran, Phys. Rev. B 52, 10673 (1995)

17. I.S. Masymov, L. F. Marsal, M. A. Ustantsev, and J. Pallares, Opt. Commun. 248, $469(2005)$

18. I.S. Masymov, L. F. Marsal, and J. Pallares, Opt. and Quant. Electron. 37, 161 (2005)

19. A. Cicek and B. Ulug, Opt. Commun. 281, 3924 (2008)

20. N. Bonod, E. Popov, and M. Nevière, Opt. Commun. 244, 389 (2005)

21. J.J. Bonnefois, G. Guida, A. Priou, M. Nevière, and E. Popov, J. Opt. Soc. Am. A 23, $842(2006)$

22. N. Ahözbek and S. John, Phys. Rev. E 57, 2287 (1998)

23. N. C. Panoiu, M. Bahl, and R. M. Osgood, Opt. Lett. 28, 2503 (2003)

24. V. Lousse and J. P. Vigneron, Phys. Rev. E 63, 027602 (2001)

25. B. Maes, P. Bienstman, and R. Baets, J. Opt. Soc. Am. B 22, 613 (2005)

26. G. Van der Sande, B. Maes, P. Bienstman, J. Danckaert, R. Baets, and I. Veretnicoff, Opt. Expr. 13, 1544 (2005)

27. J. E. Sipe, N. A. R. Bhat, P. Chak, and S. Pereira, Phys Rev. E 69, 016604 (2004)

28. S. N. Volkov and J. E. Sipe, Phys. Rev. E 70, 066621 (2004).

29. Y.R. Shen, Principles of nonlinear optics, John Wiley \& Sons Eds., 1984 
Figure 1. Schemes of the 2D-PC (A) and of its unit cell (B). Dashed lines in figure A correspond to the tilted 1D-PC's giving rise to the 2D-PC. OZ and OZ' are orthogonal to these gratings.

Figure 2. Configuration of the read and nth order diffracted wavevectors.

Figure 3. Grating period $(\Lambda)$ and normalized mean local intensity $\left(I_{L}\right)$ plotted as a function of the filling factor $\left(e_{1} / \Lambda\right)$ for the lower and upper band edge of the 2D-PC in figures $\mathrm{A}$ and $\mathrm{B}$ respectively. Note that the band edge wavelength is the same in figures A and B so that the band-centre wavelengths are different in the two calculations.

Figure 4. Reflectivity and normalized mean local intensity $\left(I_{L}\right)$ spectra.

Figure 5. (color online) Local intensity repartition in the 2D-PC for the upper band edge wavelength.

Figure 6. Input and output beams (A) and inside wavevectors (B) in degenerate four-wave mixing in the 2D-PC. The grating wave vectors are only represented in the case of the -1 order of diffraction of the forward pump wave.

Figure 7. Phase conjugate reflectivity spectra of the 2D-PC for different incidence angles of the signal beam. The phase conjugate reflectivity is plotted for the signal beam (continuous line) and the -1 and +1 diffracted orders (dashed and dotted lines respectively).

Figure 8. Comparison of the phase conjugate reflectivity spectra calculated for the $1 \mathrm{D}$ and 2D-PC's. Only the signal phase conjugate reflectivity is plotted for the 2D-PC. The phase conjugate reflectivity of the two diffracted signal beams which are of the same magnitude are not shown in the figure.

Figure 9. Relative third and fifth order correction to the normalized mean local intensity plotted as a function of the signal wavelength. 

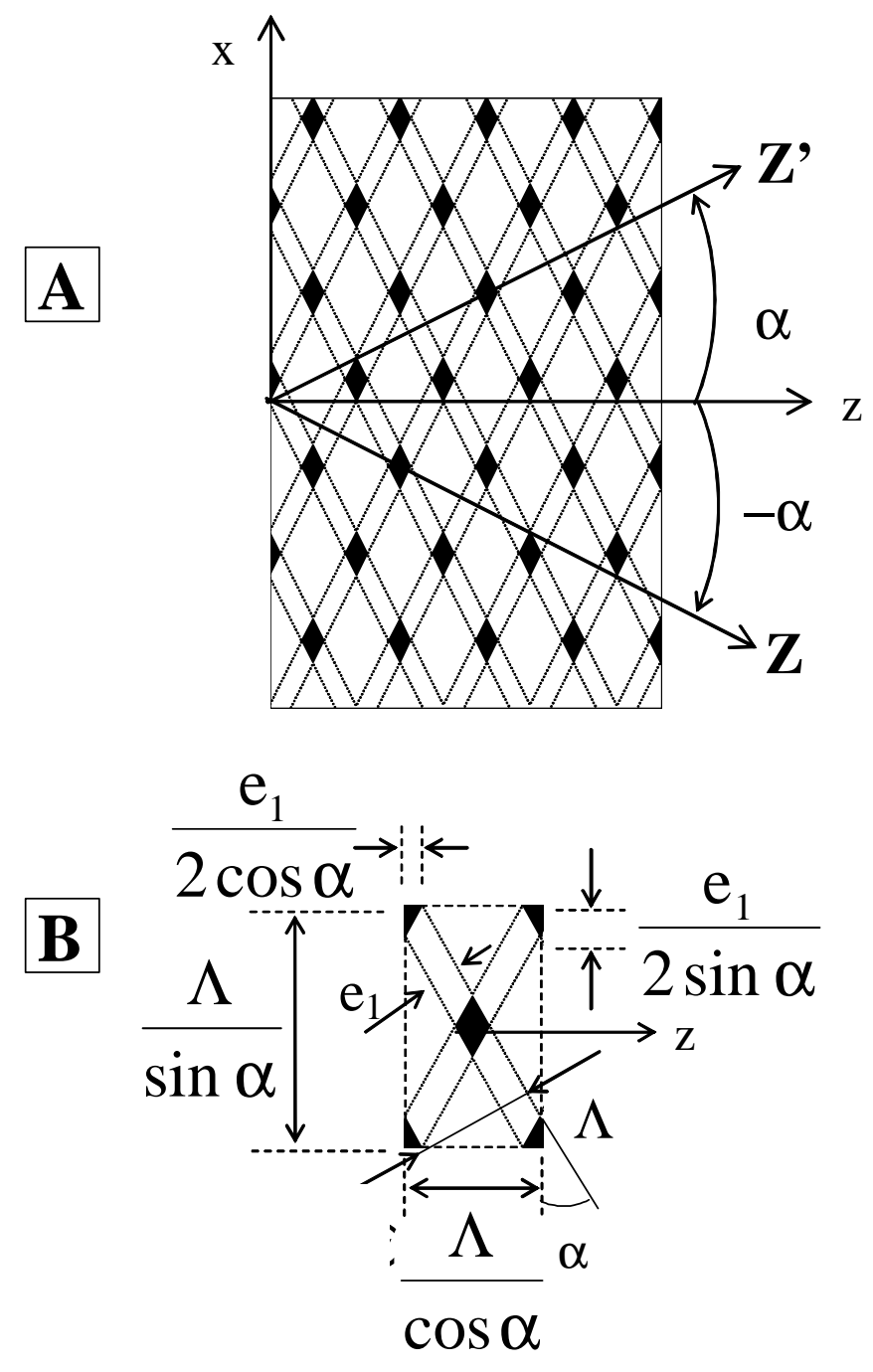

Figure 1 


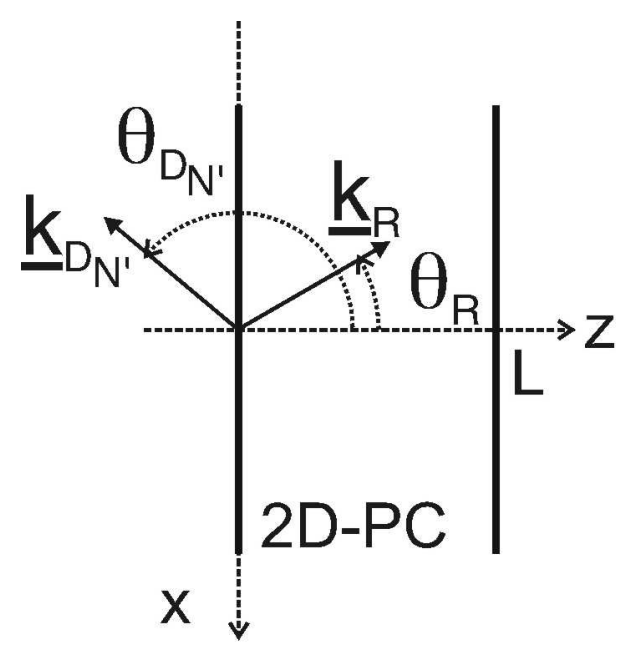

Figure 2 


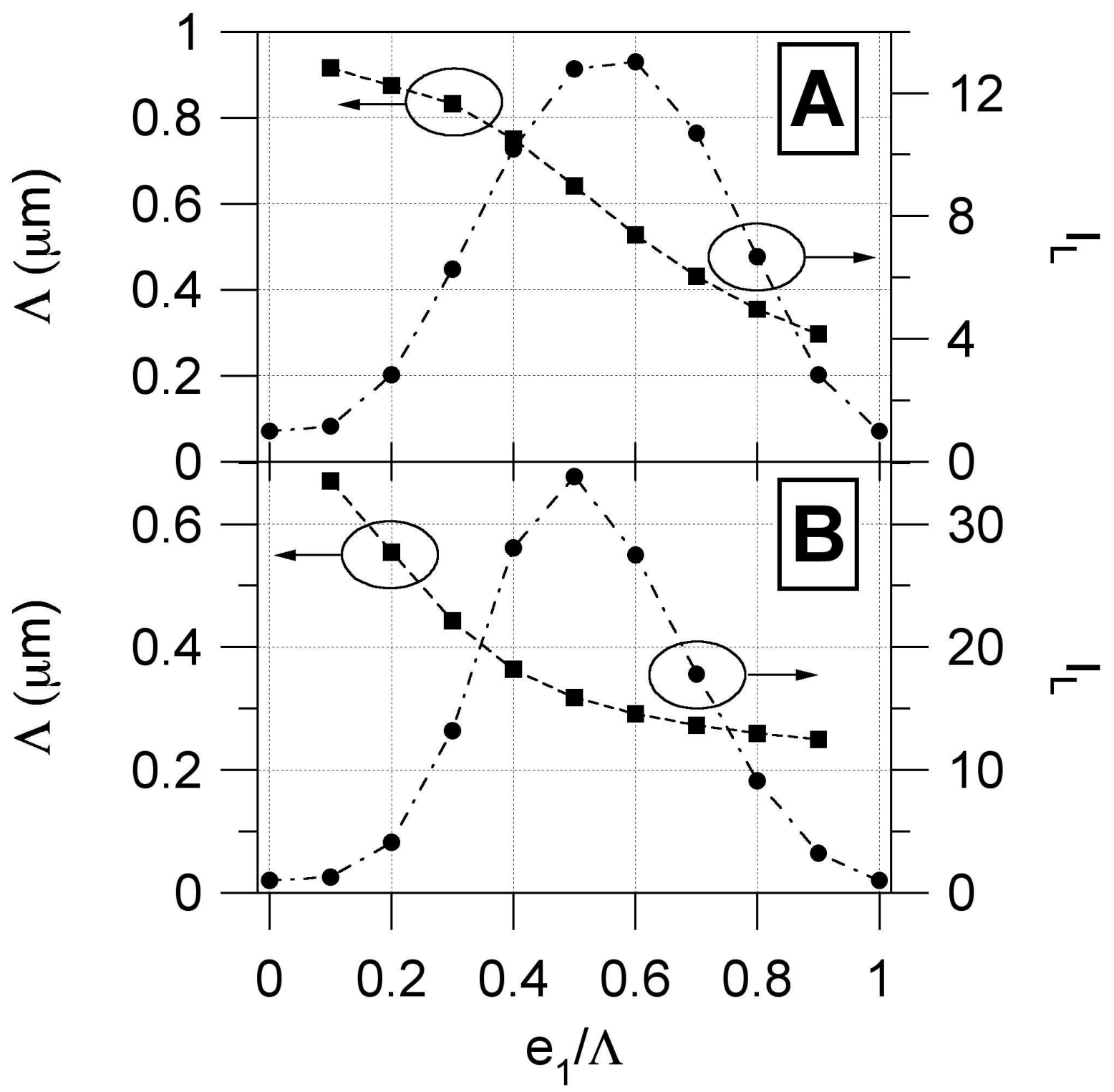

Figure 3 


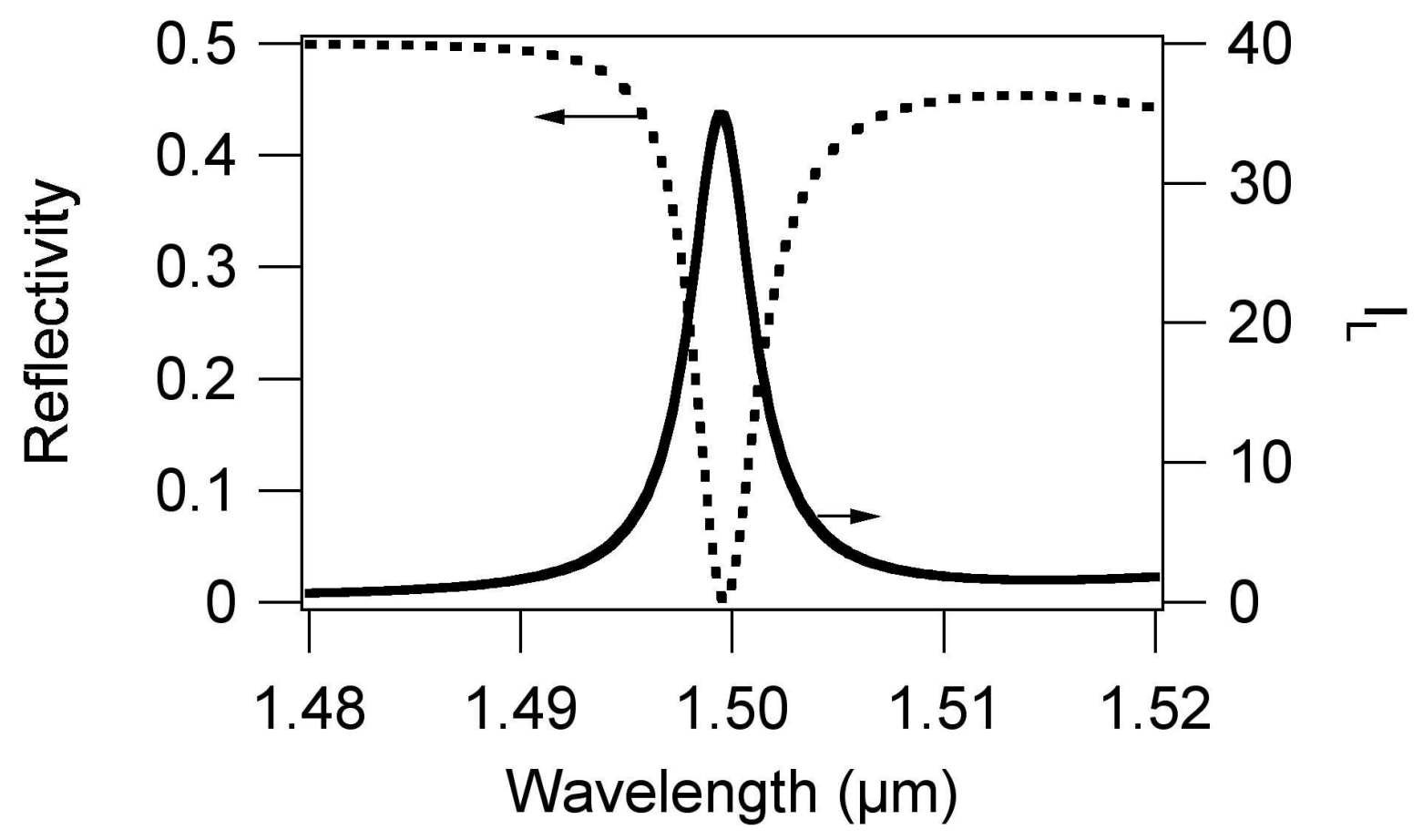

Figure 4 

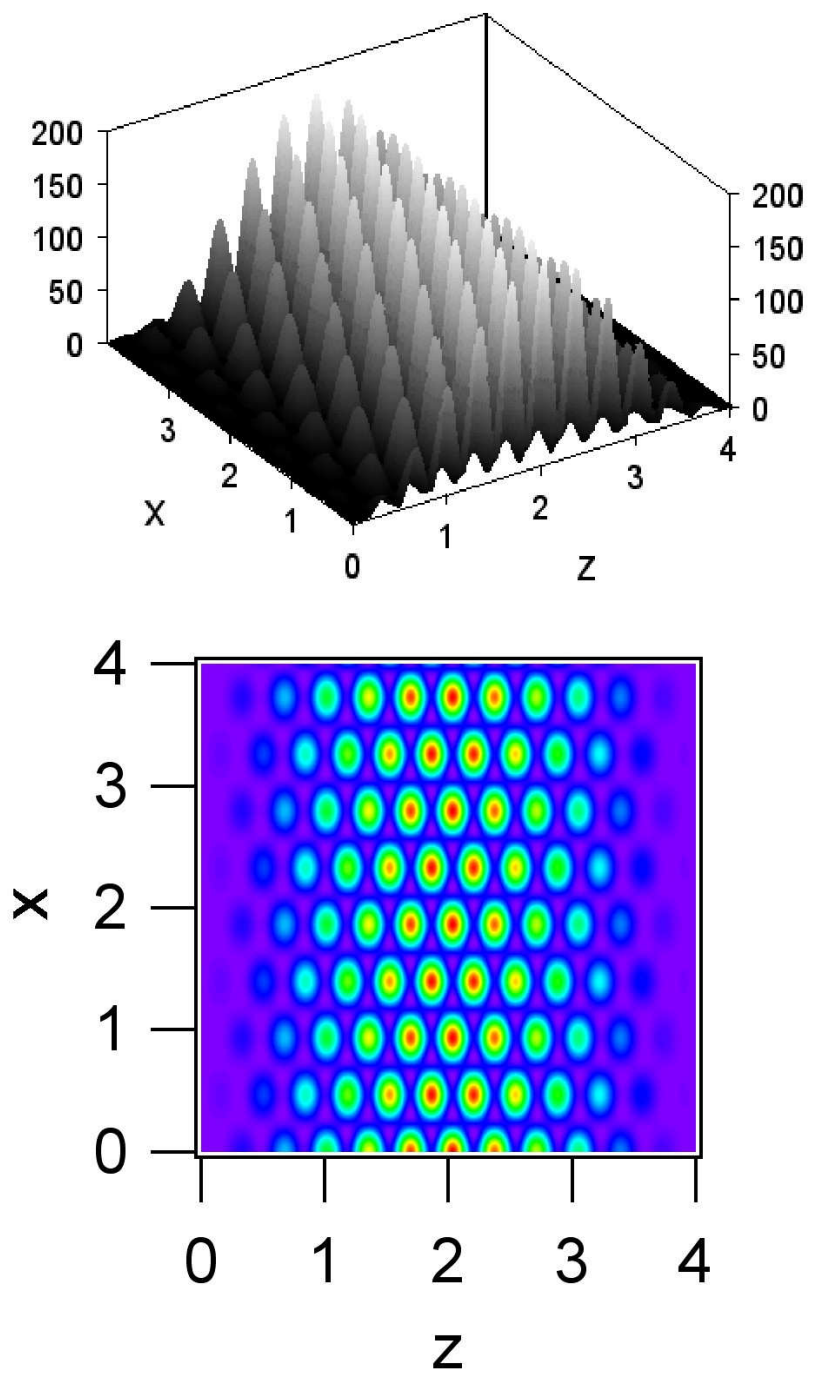

Figure 5 

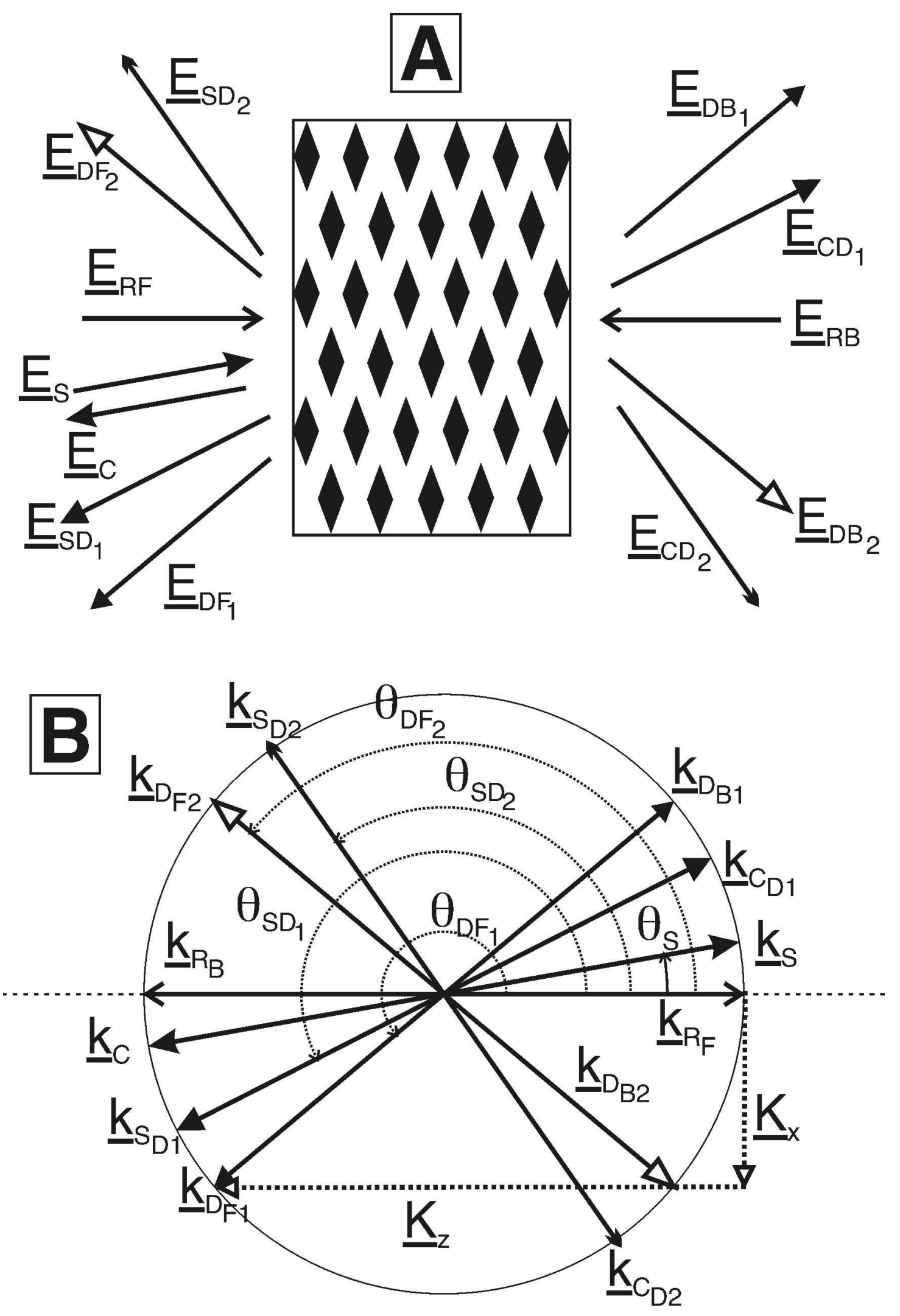

Figure 6 


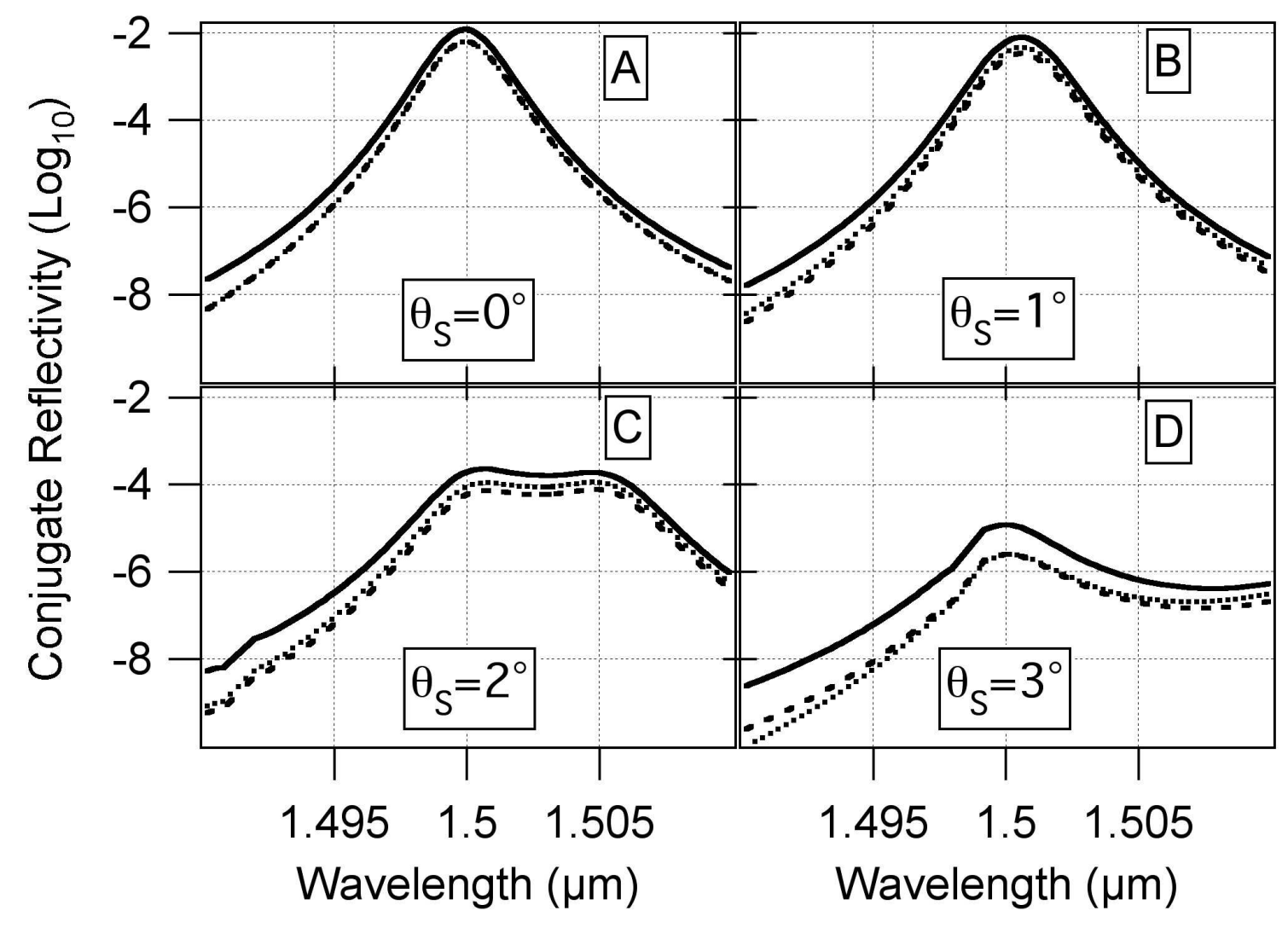

Figure 7 


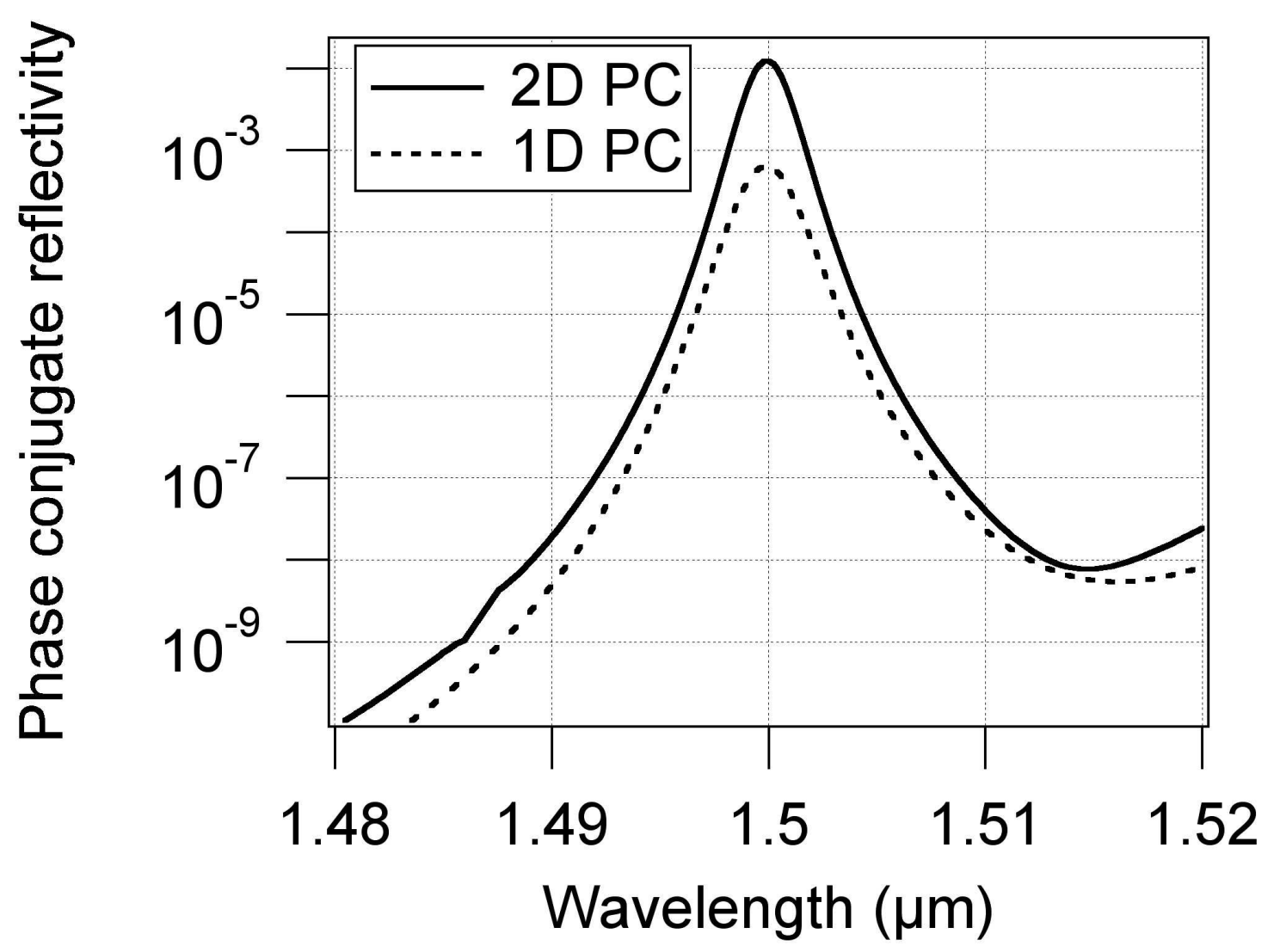

Figure 8 


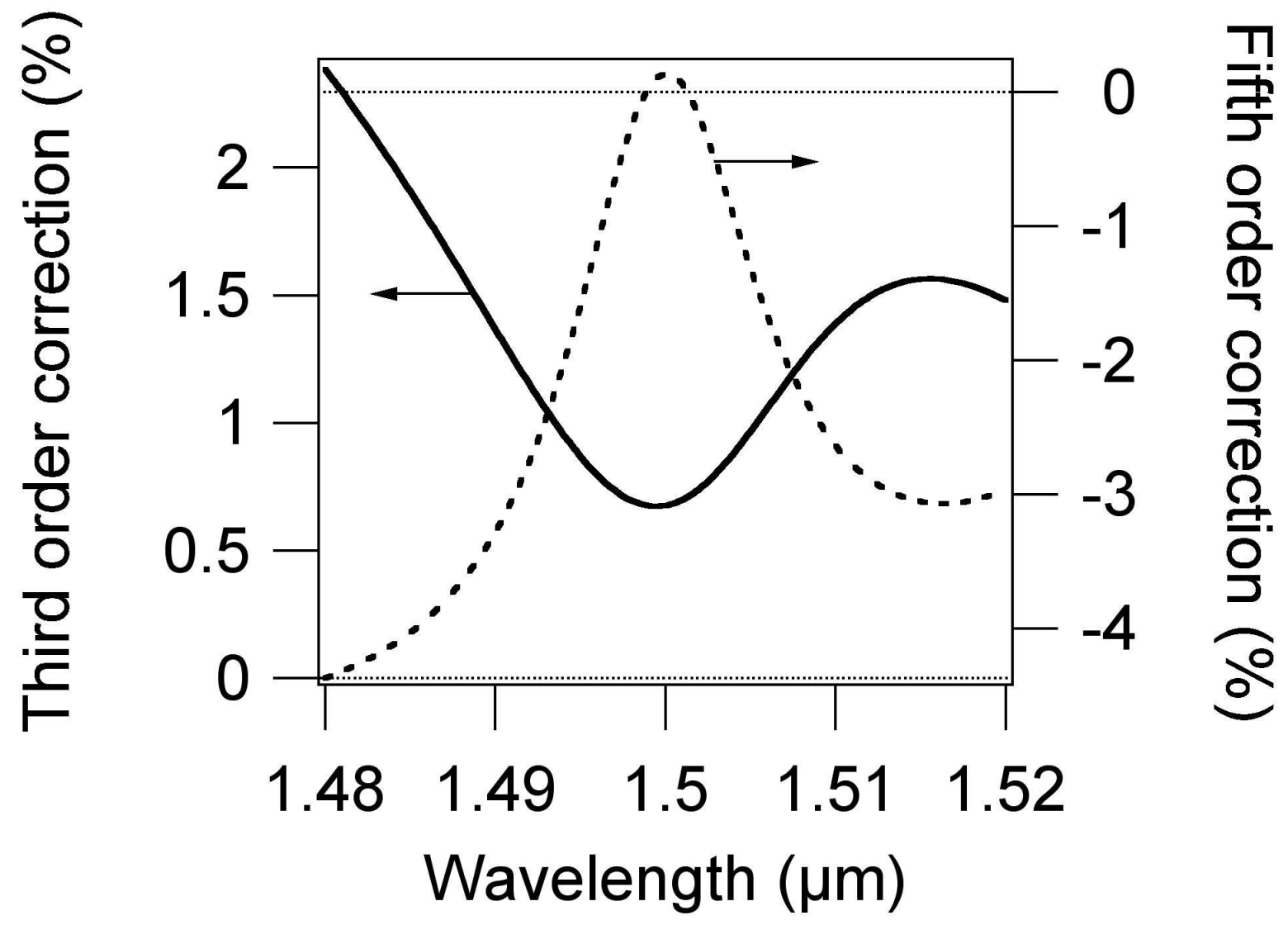

Figure 9 